\title{
High prevalence of hepatitis $B$ virus dual infection with genotypes $A$ and $G$ in HIV-1 infected men in Amsterdam, the Netherlands, during 2000-2011
}

Antoinette C van der Kuyl ${ }^{1 *}$, Fokla Zorgdrager ${ }^{1}$, Boris Hogema ${ }^{2}$, Margreet Bakker ${ }^{1}$, Suzanne Jurriaans ${ }^{3}$, Nicole KT Back ${ }^{3}$, Ben Berkhout ${ }^{1}$, Hans L Zaaijer ${ }^{2,3}$ and Marion Cornelissen ${ }^{1}$

\begin{abstract}
Background: Hepatitis B virus (HBV) is divided into 8 definite $(A-H)$ and 2 putative $(I, J)$ genotypes that show a geographical distribution. HBV genotype $G$, however, is an aberrant genotype of unknown origin that demonstrates severe replication deficiencies and very little genetic variation. It is often found in co-infections with another HBV genotype and infection has been associated with certain risk groups such as intravenous drug users and men having sex with men (MSM). We aimed to estimate the prevalence of HBV-G in the Netherlands by analysing samples from HBV-positive patients visiting the Academic Medical Center in Amsterdam.
\end{abstract}

Methods: Ninety-six HBV-infected patients, genotyped as HBV-A or HBV-G infected, were retrieved from the clinical database. Blood plasma samples were analysed with a newly-developed real-time PCR assay that detects HBV-A and HBV-G. For three patients, the HBV plasma viral load ( $\mathrm{pVL}$ ) of both genotypes was followed longitudinally. In addition, three complete genomes of HBV-G were sequenced to determine their relationship to global HBV-G strains.

Results: Ten HBV-G infections were found in the selected Dutch patients. All concerned HIV-1 infected males with HBV-A co-infection. Dutch HBV-G strains were phylogenetically closely related to reference HBV-G strains.

Conclusions: In this study, HBV-G infection in the Netherlands is found exclusively in HIV-1 infected men as co-infection with HBV-A. A considerable percentage (37\%) of men infected with HBV and HIV-1 are actually co- infected with two HBV genotypes.

\section{Background}

Hepatitis B virus (HBV), the prototype member of the Hepadnaviridae, can cause chronic hepatitis, cirrhosis and ultimately hepatocellular carcinoma. HBV is currently divided into eight confirmed genotypes that differ $>8 \%$ from each other at the nucleotide level: A to $\mathrm{H}$ (for a review, see: [1]). In addition, a novel genotype I has been reported from South-East Asia [2-5] and a candidate tenth genotype J was suggested [6]. Most genotypes can be split into subgenotypes that differ $>4 \%$ from each other, such as

\footnotetext{
* Correspondence: a.c.vanderkuyl@amc.uva.nl

'Laboratory of Experimental Virology, Department of Medical Microbiology, Center for Infection and Immunity Amsterdam (CINIMA), Academic Medical Center of the University of Amsterdam, Meibergdreef 15, Amsterdam 1105, $\mathrm{AZ}$, Netherlands

Full list of author information is available at the end of the article
}

HBV-A1 and HBV-A2 [7]. HBV-A2 is the most prevalent HBV variant in Western Europe and North America [7].

HBV genotype G (HBV-G), originally described as a mutated HBV variant that emerges during chronic HBV infection [8,9], was recognized as a separate genotype in 2000 [10]. HBV-G is distinct from other HBV genotypes in having a 36-nucleotide insertion at the $5^{\prime}$ end of the core protein gene, which adds 12 amino acids to the protein and interferes with core protein expression and virion secretion [11-13]. In addition, one or two stopcodons are usually present in the precore region that preclude $\mathrm{HBeAg}$ expression $[10,14]$. Altogether, these characteristics hamper virus replication and make HBV-G mono-infection a rarely observed event [14-16]. Apparently, HBV-G replication can be rescued by co-infection with another HBV genotype, and such dual infections have been observed repeatedly with HBV genotype A in Japan $[17,18]$, the USA

\section{Ciomed Central}

(c) 2013 van der Kuyl et al.; licensee BioMed Central Ltd. This is an open access article distributed under the terms of the Creative Commons Attribution License (http://creativecommons.org/licenses/by/2.0), which permits unrestricted use distribution, and reproduction in any medium, provided the original work is properly cited. 
$[19,20]$, Spain [21] and Canada [22], with HBV genotypes A, C or D in Germany [23,24], with HBV genotype $\mathrm{H}$ in Mexico [25], and with HBV genotype F in Argentina [26]. In fact, rescue by another genotype can be very effective, even to the point where HBV-G outcompetes the coinfecting strain $[9,17,19]$. This phenomenon has also been observed in humanized mouse models $[27,28]$. Infections with HBV-G are especially prevalent in specific risk groups such as human immunodeficiency virus type 1 (HIV-1) infected men having sex with men (MSM) and intravenous drug users [18,21,22,25,26,29-31]. HBV-G strains isolated worldwide show very little genetic variation compared to other HBV genotypes [10]. There are indications that mixed HBV infections that include an HBV-G strain are associated with increased liver fibrosis $[27,28,30]$, suggesting that patients infected with HBV-G should be monitored more closely. Also, response to antiviral treatment has been reported to be less effective in patients co-infected with HBV-G as compared to single genotype infections [23].

The prevalence of HBV-G infections in most countries in northern Europe, including the Netherlands, is not known. Therefore, we determined the occurrence of HBV-G infections and its association with HBV-A in patients visiting our academic hospital in Amsterdam, the Netherlands during 2000-2011. HBV-A is the most prevalent, endemic HBV genotype in the Netherlands and is found especially among MSM [32]. In addition, we determined the longitudinal plasma viral load $(\mathrm{pVL})$ changes of $\mathrm{HBV}$ in genotypes $\mathrm{A}$ and $\mathrm{G}$ in three dually infected patients. The complete genomes of three HBV genotype $G$ strains were sequenced to analyse their genetic relatedness to genotype $G$ strains isolated in other countries worldwide.

\section{Methods}

\section{Patient materials}

A total of 1674 patients tested HBsAg positive in the AMC (Amsterdam, the Netherlands) during 2000-2011, of which 1008 were also tested for HIV-1 infection (HBV tests requested from outside the AMC, but performed here were not included in the numbers). Of those $1008 \mathrm{HBV}$ positive patients, 211 were found to be also HIV-positive. Patients receiving anti-HBV treatment in whom therapy failure was observed and drug resistance was thus suspected $(\mathrm{N}=343)$, HBV genomic fragments were amplified, sequenced, genotyped and analysed for drug-resistance mutations. For this study, we selected AMC patients from the database of the AMC Laboratory of Clinical Virology (Department of Medical Microbiology) that previously were found to be infected with HBV genotype A or G. HBV genotyping was done with an in-house sequencing assay. The Laboratory of Clinical Virology participates in the Quality Control for
Molecular Diagnostics Hepatitis B virus Genotype EQA Programme [33] to ensure adequate performance of this genotyping assay. A total of 96 patient entries met these criteria and had sample availability; 91 had been diagnosed as HBV genotype A and 5 as HBV genotype G. Twenty-seven were HIV-positive (all males), 54 were HIV-negative and 15 had an unknown HIV status. In total, sixty-nine patients were male, and 27 were female. Of the 69 included men, 25 originated from the Netherlands; the others were from Suriname $(\mathrm{N}=11)$, Africa $(\mathrm{N}=8)$, other European countries $(\mathrm{N}=5)$, or of unknown or other origin $(\mathrm{N}=20)$. Of the 27 female patients, 6 were from the Netherlands, 15 from Suriname, 4 from Africa, and for 2 the country of origin was unknown.

\section{Real-time PCR assay}

Viral DNA was isolated from blood plasma samples with the QIAamp UltraSens Virus Kit (QIAGEN, the Netherlands). Single-tube real-time PCR reactions that specifically quantify HBV genotype A or G DNA were developed in the HBV X-protein gene and analysed on an ABI PRISM 7000 Sequence Detection System (Applied Biosystems, Foster City, CA, USA) using the primers and probes shown in Table 1 . Primers and probes were highly specific for the targeted HBV genotype (either A or G). Cross-reactivity was not detected as the primers and probes were able to amplify 5 copies of the targeted genotype in a background of up to $10^{8}$ copies of the nontargeted genotype. All subgenotypes of HBV-A can be quantified with this assay. The lower limit of detection is 5 copies per reaction $(=1000$ copies $/ \mathrm{ml})$.

\section{HBV-G genome sequencing}

HBV genotype $G$ genome fragments were amplified from viral DNA isolated from plasma samples and directly sequenced with the BigDye Terminator cycle sequencing kit (Applied Biosystems, Foster City, CA,

Table 1 Primers and probes used in the HBV-A and HBVG genotyping assay

\begin{tabular}{lll}
\hline Primer & $\begin{array}{l}\text { Position in } \\
\text { HBV genome* }\end{array}$ & Sequence $\left(\mathbf{5}^{\prime} \rightarrow \mathbf{3}^{\prime}\right)$ \\
\hline 5'HBV-A-TM3_2 & $1585-1606$ & TTC GCT TCA CCT CTG CAC GTT G \\
3'HBV-A-TM3 & $1669-1647$ & $\begin{array}{l}\text { CCA AGA GTC CTC TTA TGT AAG } \\
\text { AC }\end{array}$ \\
3'HBV-A-probe- & $1636-1613$ & $\begin{array}{l}\text { ATC TGA TGG GCG TTC ACG GTG } \\
\text { TMC }\end{array}$ \\
5'HBV-G-TM3_2 & $1585-1606$ & $\begin{array}{l}\text { TC GCT TCA CCT CTG CAC GTT A } \\
\text { 3'HBV-G-TM3 }\end{array}$ \\
$\begin{array}{ll}\text { CCA AGA GTC CTC TTA TAT AAC } \\
\text { 3'HBV-G-probe- }\end{array}$ & $1636-1613$ & $\begin{array}{l}\text { TG ATG ATG AGA GGT GTT CAT GGC } \\
\text { TM3_2 }\end{array}$ \\
\hline
\end{tabular}

*Numbering starting at the EcoR1 site. 
USA). Electrophoresis and data collection were performed on an ABI PRISM 3100 genetic analyser (also from Applied Biosystems). Sequences were assembled with CodonCode Aligner [34], and aligned with reference HBV sequences from the NCBI nucleotide database [35] using ClustalW implemented in BioEdit Sequence Alignment Editor version 7.0.9 [36]. GenBank accession numbers for the full-length genotype $G$ sequences are: KF767450, KF767451, and KF767452.

\section{Ethics}

No specific approval by the Institutional Review Board (IRB) of the Academic Medical Center (AMC) of the University of Amsterdam was needed for this study in the Netherlands, because the body materials for this study were collected under medical treatment, and can be used for further scientific research when the patients have given consent and samples are analysed anonymously, as was the case here (Code of Conduct as implemented by the Committee on Regulation of Research COREON of the Netherlands Epidemiological Society and the Dutch Federation of Biomedical Scientific Societies that is followed by the IRB of the AMC).

\section{Results}

\section{Prevalence of HBV-A and -G in Amsterdam}

The prevalence of $\mathrm{HBV}$ genotypes as determined by the Laboratory of Clinical Virology in HBV-infected patients visiting the AMC is shown in Figure 1. Genotypes D (29\%) and A (27\%) were the most prevalent, followed by genotypes E (16\%), C (15\%) and B (12\%). Genotype F or $\mathrm{G}$ infections are uncommon $(\leq 1 \%)$.

A total of 96 patients with HBV infection genotype A or $G$ infection for whom samples were available were assessed for the presence of single (A or G) or A/G dual
HBV infection with a single-tube real-time PCR assay that specifically quantifies these genotypes. Patient characteristics and results are summarized in Table 2. All female patients $(\mathrm{N}=27)$ were found to be singly infected with HBV-A. Of the 69 male patients, 55 were infected with HBV-A only, but 10 were dually infected with both HBV-A and -G. According to the clinical database all 10 dual infected patients were infected with the HBV subgenotype A2. In four male patients, the HBV pVL was below the detection limit of the assay. None of the patients exhibited a single infection with HBV-G; all 5 patients previously diagnosed as HBV genotype $G$ also carried genotype A. Five patients previously diagnosed as genotype A also carried genotype G. Strikingly, all ten dually HBV-A/G infected patients were also infected with HIV-1. In contrast, none of the HIV-1 negative patients (42 males and 27 females) were dually HBV infected. So, HBV-A/G dual infection is significantly associated with HIV-1 infection (two-tailed Fisher's exact test, $\mathrm{p}<0.0001$ ). In total, $37 \%$ of HBV/HIV-1 infected patients harboured two HBV genotypes. All ten dually $\mathrm{HBV}$ infected patients were $\mathrm{HBeAg}$ positive at the time of testing with the AxSYM ${ }^{\circ} \mathrm{HBe}$ Assay (Abbott Diagnostics, Lake Forest, Ill, USA), suggestive of infection with a precore protein producing strain [19], such as HBV-A [21]. The majority of HIV-1 infected males were of Dutch ethnicity $(13 / 23)$, with an equal distribution between those singly $(7 / 13)$ or dually HBV (6/10) infected. For the other HIV-1 infected patients the origin was either unknown $(4 / 23)$ or varied widely $(6 / 23$, from the Dominican Republic, Ecuador, Dutch Antilles, Ethiopia, and Germany). Of the HBV-A/G dually infected patients, five had unknown risk factors for infection, 1 reported heterosexual contacts as a risk factor, and four were labelled as MSM in the database.

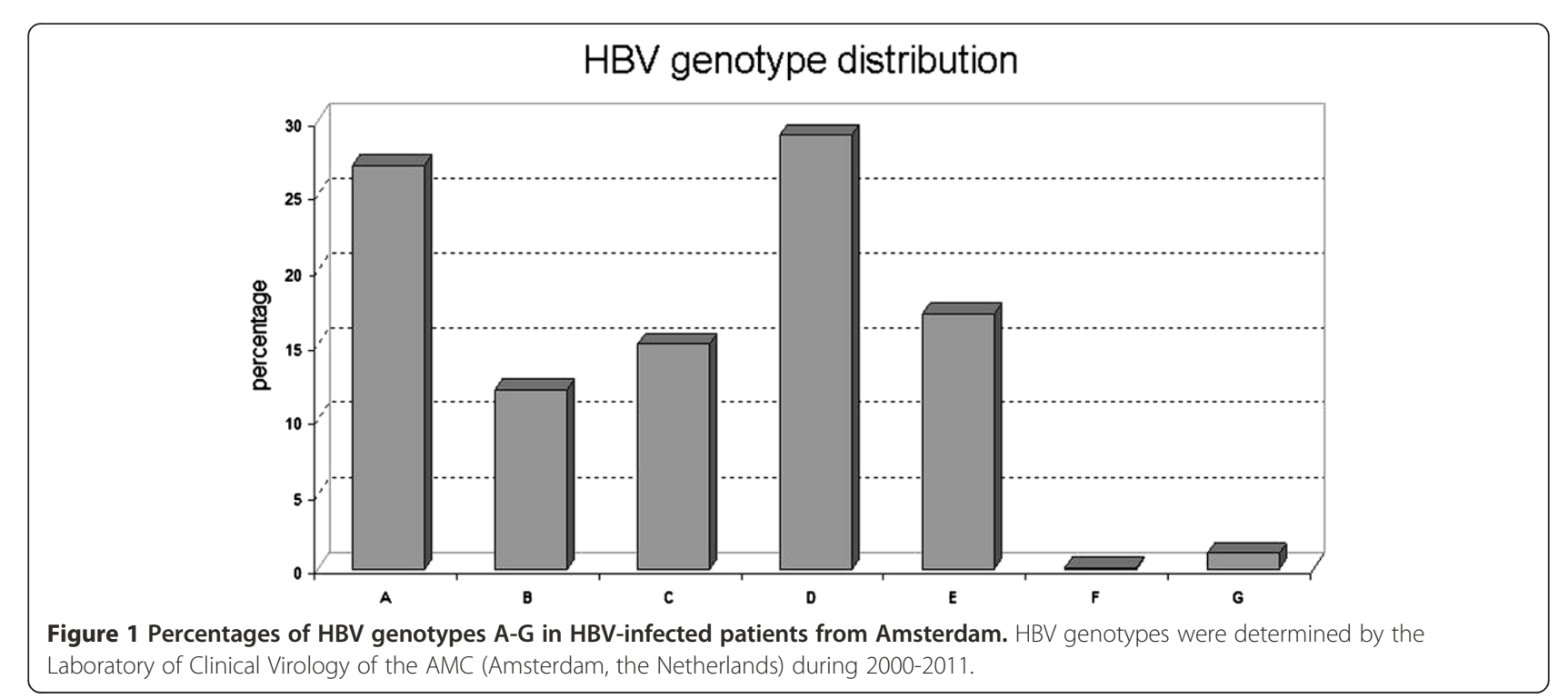


Table 2 Patient characteristics and results

\begin{tabular}{llllll}
\hline Gender & N & HIV-1 status & & HBV real-time PCR \\
\hline Male & 69 & Negative: & $\mathrm{N}=32$ & HBV-A: & $\mathrm{N}=55$ \\
& & Positive: & $\mathrm{N}=27$ & HBV-A + HBV-G: & $\mathrm{N}=10$ \\
& & Unknown: & $\mathrm{N}=10$ & Undetectable: & $\mathrm{N}=4$ \\
Female & 27 & Negative: & $\mathrm{N}=22$ & HBV-A: & $\mathrm{N}=27$ \\
& & Positive: & $\mathrm{N}=0$ & HBV-A + HBV-G: & $\mathrm{N}=0$ \\
& & Unknown: & $\mathrm{N}=5$ & Undetectable: & $\mathrm{N}=0$ \\
Total & \multirow{2}{*}{96} & Negative: & $\mathrm{N}=54$ & HBV-A: & $\mathrm{N}=82$ \\
& & Positive: & $\mathrm{N}=27$ & HBV-A + HBV-G: & $\mathrm{N}=10$ \\
& & Unknown: & $\mathrm{N}=15$ & Undetectable: & $\mathrm{N}=4$ \\
\hline & & & & &
\end{tabular}

Thus, HBV-G infection in Dutch patients is strongly associated with HBV-A and HIV-1 co-infection, which likely explains the male-specificity in this analysis, as the majority of HIV-1 infected patients in the Netherlands are male $(80 \%$ in 2011), of which $73 \%$ belong to the MSM risk group [37]. These results suggest that HBV-G is primarily circulating among MSM in the Netherlands.

To assess the possibility that both HBV-G and HIV-1 circulate in a certain subgroup of patients, as well as investigating a potential simultaneous transmission of both viruses we analysed HIV-1 polymerase sequences that were available for 6 out $10 \mathrm{HIV}-1 / \mathrm{HBV}-\mathrm{A} / \mathrm{G}$ infected patients and $10 \mathrm{HIV}-1 / \mathrm{HBV}-\mathrm{A} 2$ infected patients that were not infected with HBV-G. A phylogenetic tree was generated of these 16 sequences plus reference sequences of HIV-1 subtypes A-K with the neighbour-joining option in MEGA5 based upon a Kimura 2-parameter distance matrix [38]. The NJ tree suggested no significant relationship between the HIV-1 polymerase sequences of the $6 \mathrm{HBV}-\mathrm{A} / \mathrm{G}$ dual infected patients (not shown) suggesting that transmission of HIV-1 and HBV-G are independent events.

\section{Complete HBV-G genome analysis}

To investigate whether HBV-G strains circulating in the Netherlands are similar to global HBV-G strains, the DNA genome of three virus isolates (from patients 7, 23 and 27, all of Dutch ethnicity) were amplified and completely sequenced. The sequences demonstrated the characteristic 36-nucleotide insertion at the $5^{\prime}$ end of the core protein gene and two stopcodons in the precore region that preclude $\mathrm{HBeAg}$ expression. Also, a fourth, but incompletely sequenced HBV-G genome from patient 26 (originating from the Dominican Republic, but infected in the Netherlands, where he presented with an acute HBV infection) showed high sequence similarity with the other Dutch strains (not shown). HBV-G strains of patients 7 and 27 were closely related to each other and to an HBV-G strain originating from a Dutch blood donor with a HBV-G mono-infection [14]. The virus isolates of both patients showed resistance mutations against 3TC in the RT region (L180M and M204V).

All three completely sequenced Dutch HBV-G strains clustered with HBV-G reference sequences in a phylogenetic analysis (Figure 2). Genetic distances between the Dutch HBV-G genomes and reference HBV-G sequences were extremely low, and ranged between $0.002-$ 0.008 for the full-length sequences. This is in line with the low genetic diversity of less than $0.5 \%$ detected for all HBV-G strains isolated worldwide [39].

For the other 7 patients, a 359 nucleotide fragment spanning the precore/core region of HBV-G was amplified and sequenced. All 7 isolates contained the typical 36-nucleotide insertion as well as the two stopcodons, and clustered with HBV-G sequences.

\section{HBV-A and -G plasma viral load}

For three HBV-A/G dually infected patients (nos. 7, 12 and 23), serial blood plasma samples were available for a longitudinal analysis. In all three patients, both genotypes were already present in the first available sample, so that the order of infection could not be established. A longitudinal analysis of the pVL of each HBV genotype was performed with the real-time PCR assay (Figure 3). All three patients were also HIV-1 infected, and treated with antiretroviral therapy, including lamivudine (3TC) and tenofovir (TDF) that target both the HIV-1 and the HBV RT enzymes. In general, the pVL of HBV-A and HBV-G follow a similar course in these patients, increasing or decreasing in parallel.

In patient 7 , the HBV-G pVL exceeded the HBV-A $\mathrm{pVL}$ considerably ( $>10^{5}$ difference) at all time-points over a 4-year period (Figure 3A). The HBV-A and HIV-1 pVL decreased significantly after the start of an ART regimen containing $3 \mathrm{TC}$ in 2001, and remained close to or below the detection limit of the assay afterwards for HBV-A (Figure 3A). 3TC drug resistance occurred in the HBV-G strain, and its pVL rebounded after an initial drop. Subsequent initiation of TDF treatment (in addition to 3TC) resulted in a significant decrease in the HBV-G pVL.

In patient 12 , the HBV-G pVL exceeded the HBV-A $\mathrm{pVL}$ at least tenfold over a 42 month period, except in the first sample measured (Figure 3B). A period without ART resulted in an increase in HBV pVL of both genotypes that decreased again with the restart of ART that included TDF (Figure 3B).

In patient 23 , the $\mathrm{pVL}$ of the two genotypes was comparable over an 8-year period, despite dramatic fluctuations due to therapy changes (Figure 3C). The HBV-A and -G pVL also decreased sharply with the start of an ART regimen that contained both 3TC and TDF. However, mutations associated with 3TC resistance (L180M and M204V) developed three years later in both 


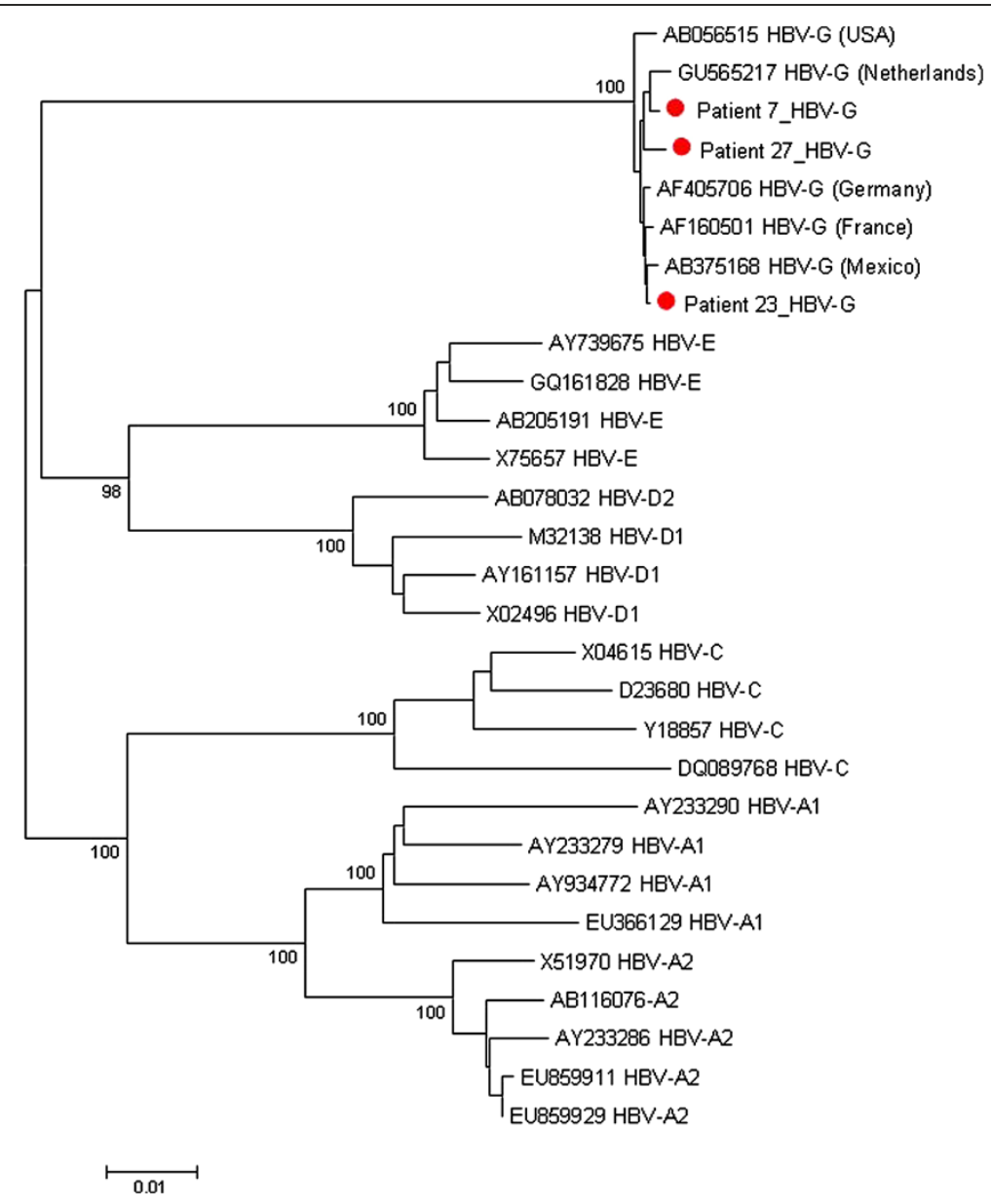

Figure 2 Phylogenetic analysis of full-length HBV sequences. The neighbour-joining tree was generated with the MEGA5 software package [38] and shows the relationship between three novel, full-length HBV-G strains from the Netherlands (indicated by red circles) and reference sequences of HBV genotypes A1, A2, C, D1, D2 and G obtained from the NCBI nucleotide database [35]. Distances were estimated with the Kimura 2-parameter method, and bootstrap resampling was done with 1000 replicates. The accession number of the reference sequences is indicated together with the HBV genotype.

genotypes. A regimen containing TDF was subsequently installed, which lowered both HBV pVLs to just above the detection limit of the assay.

\section{Discussion}

In a population of $96 \mathrm{HBV}$-infected patients visiting our academic hospital in Amsterdam that had been genotyped to harbour HBV-A or HBV-G, $10 \mathrm{HBV}-\mathrm{A} / \mathrm{G}$ dual infections were detected. All $10 \mathrm{HBV}-\mathrm{G}$ infections concerned men that were also infected with HIV-1. Studies from other countries report a similar association of HBV-G infection with HIV-infection and with the MSM risk group [22,25,26,30,31]. In our study, only four HBVA/G infected patients could be positively identified as MSM, for five others the risk factor was not stated. But as HBV-A is largely spreading in MSM (as is HIV-1) in the Netherlands; their HBV-G infection is most likely also due to homosexual contacts.
In contrast to the first report of HBV-G in the Netherlands that occurred as a mono-infection, the HBV-G infections detected in this study all represent co-infections with HBV-A. The initial mono-infection occurred in a Dutch male blood donor who denied risk factors for HBV infection, suggesting that HBV-G is also present outside the MSM risk group [14]. However, drug-resistance mutations were present in this HBV-G isolate, implying that the strain was most likely transmitted from an ART-treated individual.

HBV-G infections are mainly noticeable after infection with a "helper" HBV strain, here genotype A, and especially during HIV-1 co-infection that decreases HBV immune control and increases HBV replication [40]. HBV-G mono-infected patients have been reported to be $\mathrm{HBeAg}$ negative, variably $\mathrm{HBsAg}$ positive ( 2 out of 3 cases), while $\mathrm{HBcAb}$ and $\mathrm{HBsAb}$ levels can be inconsistent and/or delayed [14-16]. So, it is not always easy to 


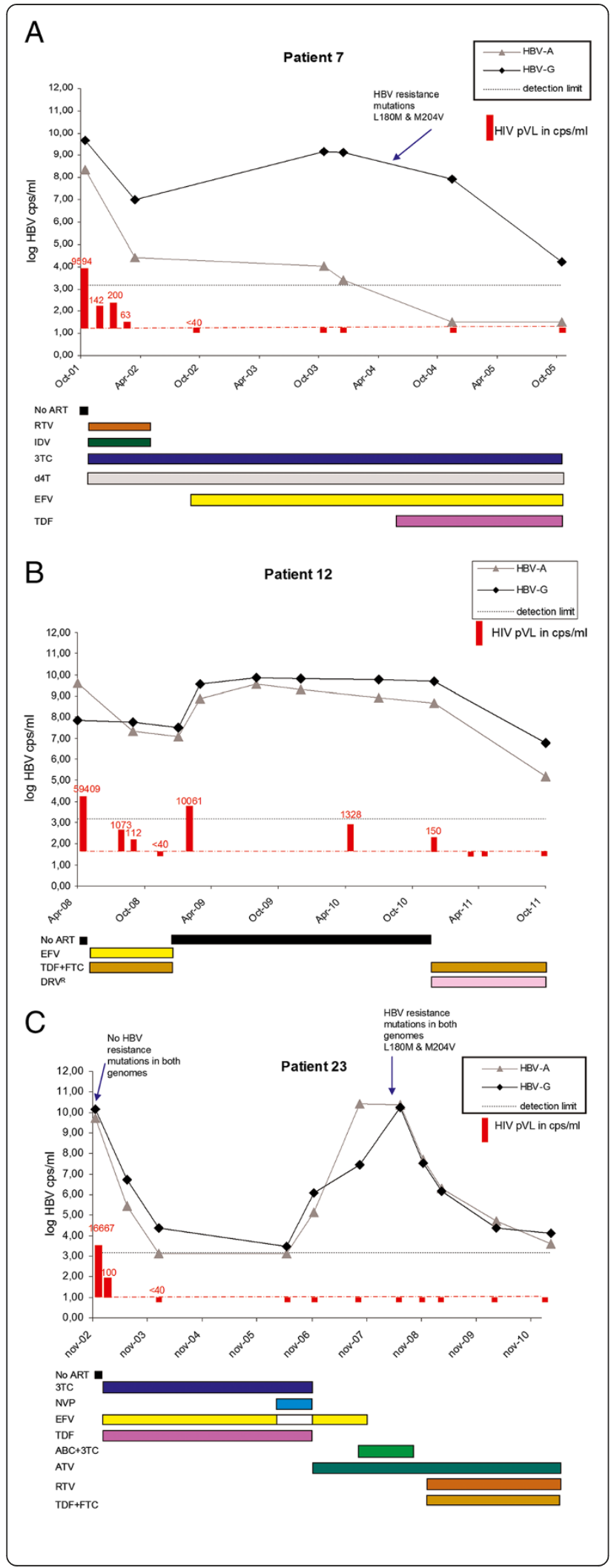

Figure 3 Longitudinal analysis of the HBV-A and $-\mathrm{G} p \mathrm{pL}$ in three male HIV-1 infected patients. Panel $\mathbf{A}=$ patient 7; panel $\mathbf{B}=$ patient 12; panel $\mathbf{C}=$ patient 23 . The HIV-1 pVL is indicated with red bars; numbers in red are HIV-1 copies/ml. ART regimens are shown below the graphs. Abbreviations: $3 T C=$ lamivudine; $A B C=$ abacavir; $A T V=$ atazanavir; $\mathrm{d} 4 \mathrm{~T}$ = stavidine; DRV = darunavir (boosted with RTV); $\mathrm{EFV}=$ efavirenz; $\mathrm{FTC}=$ emtricitabine; IDV = indiniavir; NVP = nevirapine; RTV = ritonavir; TDF = tenofovir. $3 T \mathrm{C}$ and TDF are also effective against HBV.

recognize an HBV-G mono-infected patient as being HBV positive, especially in a routine diagnostic setting, and these patients might not have been included in the current study selection. It would be interesting to examine HIV-1 infected HBV-negative MSM for HBV-G mono-infection, as both viruses are circulating in this risk group. The newly-developed real-time PCR assay has a detection limit of 5 copies per reaction, enabling the detection of the low viral DNA levels as observed in HBV-G mono-infection.

Full-length genome sequencing indicated that the newly characterized Dutch HBV-G strains are closely related to reference HBV-G strains isolated around the world. They all contained the genome peculiarities associated with HBV-G, e.g. the 36-nucleotide insertion at the $5^{\prime}$ end of the core protein gene and two stopcodons in the precore region, as did isolates from 7 other patients. This again indicates that HBV-G has no specific geographical distribution as is the case for the other HBV genotypes.

For three patients, longitudinal blood plasma samples were available. Those samples were analysed with the real-time PCR assay to measure the relative contributions of each genotype to the pVL. In one patient (no. 23), HBV-A and HBV-G replicated at a similar level. In the other two patients (no. 7 and no. 12), the HBV-G pVL exceeded the HBV-A pVL at least 10 times, in line with other reports $[9,17,19]$. The increased HBV-G load in the presence of a helper strains is also in line with the results from humanized mouse models $[27,28]$. In all three cases, the patients were already infected with both HBV genotypes and HIV-1 at the first available sampling time, so that the order of infection cannot be determined. It is possible that the infections occurred simultaneously, as $37 \%$ of the Dutch HIV-1/HBV-infected MSM were found to be co-infected with HBV-A and HBV-G, and could thus transmit multiple HBV strains simultaneously. However, in the dual HBV-A/G infections described here, the HBV$\mathrm{G}$ pVL exceeds the HBV-A pVL at least 10 times at most time points, so it is likely that HBV-G has a better chance to be transmitted. This finding of a much increased HBV$\mathrm{G} \mathrm{pVL}$ in A/G dual versus mono-infections could also explain why 5 patients were determined as being singly 
infected with HBV-G using the clinical HBV genotyping assay.

\section{Conclusions}

The prevalence of HBV genotype G in the Netherlands was investigated using real-time PCR assays. HBV-G infection was found in 10/96 individuals previously identified as infected with HBV genotype A or G. These 10 patients were all HIV-1 infected men that were also infected with a $\mathrm{HBV}$ genotype A strain, more specifically the HBV-A2 genotype that is endemic in the Netherlands. These findings suggest a strong association of HBV-G in the Netherlands with the HIV-1 infected male risk group, as has been reported from other countries.

\section{Abbreviations \\ HBV: Hepatitis B virus; HIV-1: Human immunodeficiency virus type 1; MSM: Men having sex with men; pVL: Plasma viral load.}

\section{Competing interests}

The authors declare that they have no competing interests.

\section{Authors' contributions}

ACvdK drafted the manuscript, FZ performed the experiments, $\mathrm{BH}$ was involved in sequencing, MB performed the database selections with the help of $\mathrm{SJ}$ and NKTB,BB and $H L Z$ critically revised the manuscript, and MC designed the experiments. All authors read and approved the final manuscript.

\section{Acknowledgements}

The authors thank Dr. Dominique Verhagen (Medisch Centrum Jan van Goyen, Amsterdam, the Netherlands) for sharing patient information. We are also grateful to Iris Coerman for help with the initial analyses, and to Frits Schöler (Dept. of Medical Microbiology, AMC) for additional help with databases. This work was supported in part by the Dutch Ministry of Security and Justice.

\section{Author details}

${ }^{1}$ Laboratory of Experimental Virology, Department of Medical Microbiology, Center for Infection and Immunity Amsterdam (CINIMA), Academic Medical Center of the University of Amsterdam, Meibergdreef 15, Amsterdam 1105, AZ, Netherlands. ${ }^{2}$ Department of Blood-borne infections, Sanguin, Amsterdam, Netherlands. ${ }^{3}$ Laboratory of Clinical Virology, Department of Medical Microbiology, Center for Infection and Immunity Amsterdam (CINIMA), Academic Medical Center of the University of Amsterdam, Meibergdreef 15, Amsterdam 1105AZ, Netherlands.

Received: 27 August 2013 Accepted: 12 November 2013 Published: 14 November 2013

\section{References}

1. Schaefer S: Hepatitis B virus taxonomy and hepatitis B virus genotypes. World J Gastroenterol 2007, 13:14-21.

2. Tran T, Trinh TN, Abe K: New complex recombinant genotype of hepatitis B virus identified in Vietnam. J Virol 2008, 82:5657-5663.

3. Yu H, Yuan Q, Ge SX, Wang HY, Zhang YL, Chen QR, Zhang J, Chen PJ, Xia NS: Molecular and phylogenetic analyses suggest an additional hepatitis B virus genotype "I". PLOS ONE 2010, 5:e9297.

4. Arankalle VA, Gandhe SS, Borkakoty BJ, Walimbe AM, Biswas D, Mahanta J: A novel HBV recombinant (genotype I) similar to Vietnam/Laos in a primitive tribe in eastern India. J Viral Hepat 2010, 17:501-510.

5. Tong W, He J, Sun L, He S, Qi Q: Hepatitis B virus with a proposed genotype I was found in Sichuan Province, China. J Med Virol 2012, 84:866-870.

6. Tatematsu K, Tanaka Y, Kurbanov F, Sugauchi F, Mano S, Maeshiro T, Nakayoshi T, Wakuta M, Miyakawa Y, Mizokami M: A genetic variant of hepatitis $B$ virus divergent from known human and ape genotypes isolated from a Japanese patient and provisionally assigned to new genotype J. J Virol 2009, 83:10538-10547.

7. Chudy M, Hanschmann KM, Kress J, Nick S, Campos R, Wend U, Gerlich W, Nubling CM: First WHO International Reference Panel containing hepatitis B virus genotypes A-G for assays of the viral DNA. J Clin Virol 2012, 55:303-309.

8. Bhat RA, Ulrich PP, Vyas GN: Molecular characterization of a new variant of hepatitis $B$ virus in a persistently infected homosexual man. Hepatology 1990, 11:271-276.

9. Tran A, Kremsdorf D, Capel F, Housset C, Dauguet C, Petit MA, Brechot C: Emergence of and takeover by hepatitis $B$ virus (HBV) with rearrangements in the pre-S/S and pre- $\mathrm{C} / \mathrm{C}$ genes during chronic $\mathrm{HBV}$ infection. J Virol 1991, 65:3566-3574.

10. Stuyver L, De Gendt S, Van Geyt C, Zoulim F, Fried M, Schinazi RF, Rossau R: A new genotype of hepatitis $B$ virus: complete genome and phylogenetic relatedness. J Gen Virol 2000, 81:67-74.

11. Li K, Zoulim F, Pichoud C, Kwei K, Villet S, Wands J, Li J, Tong S: Critical role of the 36-nucleotide insertion in hepatitis $B$ virus genotype $G$ in core protein expression, genome replication, and virion secretion. J Virol 2007, 81:9202-9215.

12. Gutelius D, Li J, Wands J, Tong S: Characterization of the pleiotropic effects of the genotype G-specific 36-nucleotide insertion in the context of other hepatitis B virus genotypes. J Virol 2011, 85:13278-13289.

13. Cotelesage JJ, Osiowy C, Lawrence C, DeVarennes SL, Teow S, Beniac DR, Booth TF: Hepatitis B Virus Genotype G forms core-like particles with unique structural properties. J Viral Hepat 2011, 18:443-448.

14. Zaaijer HL, Boot HJ, van SP, Koppelman MH, Cuypers HT: HBsAg-negative mono-infection with hepatitis B virus genotype G. J Viral Hepat 2011, 18:815-819.

15. Chudy M, Schmidt M, Czudai V, Scheiblauer H, Nick S, Mosebach M, Hourfar MK, Seifried E, Roth WK, Grunelt E, Nubling CM: Hepatitis B virus genotype $\mathrm{G}$ monoinfection and its transmission by blood components. Hepatology 2006, 44:99-107

16. Sayan M, Dogan C: Hepatitis B virus genotype $\mathrm{G}$ infection in a Turkish patient undergoing hemodialysis therapy. Hepat Mon 2012, 12:118-121.

17. Kato H, Orito E, Gish RG, Sugauchi F, Suzuki S, Ueda R, Miyakawa Y, Mizokami M: Characteristics of hepatitis B virus isolates of genotype $G$ and their phylogenetic differences from the other six genotypes (A through F). J Virol 2002, 76:6131-6137.

18. Shibayama T, Masuda G, Ajisawa A, Hiruma K, Tsuda F, Nishizawa T, Takahashi M, Okamoto H: Characterization of seven genotypes ( $A$ to $E, G$ and $\mathrm{H}$ ) of hepatitis $\mathrm{B}$ virus recovered from Japanese patients infected with human immunodeficiency virus type 1. J Med Virol 2005, 76:24-32.

19. Kato H, Orito E, Gish RG, Bzowej N, Newsom M, Sugauchi F, Suzuki S, Ueda R, Miyakawa $Y$, Mizokami M: Hepatitis B e antigen in sera from individuals infected with hepatitis B virus of genotype G. Hepatology 2002, 35:922-929.

20. Kato H, Gish RG, Bzowej N, Newsom M, Sugauchi F, Tanaka Y, Kato T, Orito E, Usuda S, Ueda R, Miyakawa Y, Mizokami M: Eight genotypes $(\mathrm{A}-\mathrm{H})$ of hepatitis $B$ virus infecting patients from San Francisco and their demographic, clinical, and virological characteristics. J Med Virol 2004, 73:516-521.

21. Perez-Olmeda M, Nunez M, Garcia-Samaniego J, Rios P, Gonzalez-Lahoz J, Soriano V: Distribution of hepatitis B virus genotypes in HIV-infected patients with chronic hepatitis B: therapeutic implications. AIDS Res Hum Retroviruses 2003, 19:657-659.

22. Osiowy C, Gordon D, Borlang J, Giles E, Villeneuve JP: Hepatitis B virus genotype $\mathrm{G}$ epidemiology and co-infection with genotype $A$ in Canada. J Gen Virol 2008, 89:3009-3015.

23. Erhardt A, Gobel T, Ludwig A, Lau GK, Marcellin P, van Bommel F, HeinzelPleines $U$, Adams $O$, Haussinger $D$ : Response to antiviral treatment in patients infected with hepatitis B virus genotypes E-H. J Med Virol 2009, 81:1716-1720.

24. Beggel B, Neumann-Fraune M, Doring M, Lawyer G, Kaiser R, Verheyen J, Lengauer T: Genotyping hepatitis B virus dual infections using population-based sequence data. J Gen Virol 2012, 93:1899-1907.

25. Sanchez LV, Tanaka Y, Maldonado M, Mizokami M, Panduro A: Difference of hepatitis $B$ virus genotype distribution in two groups of mexican patients with different risk factors. High prevalence of genotype $\mathrm{H}$ and G. Intervirology 2007, 50:9-15.

26. Araujo NM, Araujo OC, Silva EM, Villela-Nogueira CA, Nabuco LC, Parana R, Bessone F, Gomes SA, Trepo C, Kay A: Identification of novel recombinants 
of hepatitis B virus genotypes $F$ and $G$ in human immunodeficiency virus-positive patients from Argentina and Brazil. J Gen Virol 2013, 94:150-158.

27. Sugiyama M, Tanaka Y, Sakamoto T, Maruyama I, Shimada T, Takahashi S, Shirai T, Kato H, Nagao M, Miyakawa Y, Mizokami M: Early dynamics of hepatitis B virus in chimeric mice carrying human hepatocytes monoinfected or coinfected with genotype G. Hepatology 2007, 45:929-937.

28. Tanaka Y, Sanchez LV, Sugiyama M, Sakamoto T, Kurbanov F, Tatematsu K, Roman S, Takahashi S, Shirai T, Panduro A, Mizokami M: Characteristics of hepatitis $B$ virus genotype $\mathrm{G}$ coinfected with genotype $\mathrm{H}$ in chimeric mice carrying human hepatocytes. Virology 2008, 376:408-415.

29. Vieth S, Manegold C, Drosten C, Nippraschk T, Gunther S: Sequence and phylogenetic analysis of hepatitis B virus genotype $G$ isolated in Germany. Virus Genes 2002, 24:153-156.

30. Dao DY, Balko J, Attar N, Neak E, Yuan HJ, Lee WM, Jain MK: Hepatitis B virus genotype G: prevalence and impact in patients co-infected with human immunodeficiency virus. J Med Virol 2011, 83:1551-1558.

31. Coffin CS, Osiowy C, Myers RP, Gill MJ: Virology and clinical sequelae of long-term antiviral therapy in a North American cohort of hepatitis B virus (HBV)/human immunodeficiency virus type 1 (HIV-1) co-infected patients. J Clin Virol 2013, 57:103-108.

32. van Houdt R, Bruisten SM, Koedijk FD, Dukers NH, Op de Coul EL, Mostert MC, Niesters HG, Richardus JH, de Man RA, van Doornum GJ, van den Hoek JA, Coutinho RA, van de Laar MJ, Boot HJ: Molecular epidemiology of acute hepatitis B in the Netherlands in 2004: nationwide survey. J Med Virol 2007, 79:895-901.

33. Quality Control for Molecular Diagnostics Hepatitis B virus Genotype EQA Programme. www.QCMD.org/.

34. CodonCode Corporation. www.codoncode.com/aligner/.

35. NCBI nucleotide database. www.ncbi.nlm.nih.gov/nucleotide/.

36. BioEdit Sequence Alignment Editor version 7.0.9. www.mbio.ncsu.edu/BioEdit/ bioedit.html.

37. HIV Monitoring Foundation. http://www.hiv-monitoring.nl/index.php/ nederlands/.

38. MEGA 5 software package. www.megasoftware.net/.

39. Lindh M: HBV genotype G-an odd genotype of unknown origin. J Clin Virol 2005, 34:315-316.

40. Benhamou Y: Hepatitis B in the HIV-coinfected patient. J Acquir Immune Defic Syndr 2007, 45(Suppl 2):S57-S65.

doi:10.1186/1471-2334-13-540

Cite this article as: van der Kuyl et al: High prevalence of hepatitis B virus dual infection with genotypes $A$ and $G$ in HIV-1 infected men in Amsterdam, the Netherlands, during 2000-2011. BMC Infectious Diseases 2013 13:540

\section{Submit your next manuscript to BioMed Central and take full advantage of:}

- Convenient online submission

- Thorough peer review

- No space constraints or color figure charges

- Immediate publication on acceptance

- Inclusion in PubMed, CAS, Scopus and Google Scholar

- Research which is freely available for redistribution 